\section{Rutherford Syndrome}

Jacqueline E. van der Wal

Department of Pathology, The Netherlands Cancer Institute/Antoni van Leeuwenhoek Hospital, Amsterdam, The Netherlands

\section{Synonyms}

Gingival fibromatiosis

\section{Description}

GF, corneal opacities, retarded tooth eruption.

\section{Cross-References}

- Frictional Keratosis 